\title{
Comparative analyses of cytohistologic techniques in diagnoses of lung lesions
}

\author{
Tuladhar A 1 , Panth R ${ }^{2}$, Joshi AR ${ }^{3}$ \\ ${ }^{1}$ Department of Pathology, Kathmandu Model Hospital, Kathmandu, Nepal \\ ${ }^{2}$ Associate Professor of Pathology, National Academy of Medical Sciences, Kathmandu, Nepal \\ ${ }^{3}$ Professor of Pathology, National Academy of Medical Sciences, Kathmandu, Nepal
}

\author{
Keywords: \\ Fiberoptic bronchoscopy; \\ Lund cancer; \\ Infection; \\ Cytohistologic \\ technique
}

\begin{abstract}
Background: The use of cytological methods in the diagnosis of malignant lesions of the respiratory tract has been generally acclaimed as one of its most successful applications. Flexible fiberoptic bronchoscopy revolutionized respiratory cytology, as bronchial brushings, broncho-alveolar lavage and bronchial forceps biopsy became more easy, accessible and popular, shifting the emphasis from diagnosis of advanced malignancy in inoperable patients to the use of cytology as a first line diagnostic and management tool. Respiratory tract cytology is well established throughout the world as a diagnostic procedure in the evaluation of patient with suspected lung malignancy.
\end{abstract}

Materials and Methods: A prospective study was conducted on 50 patients divided in two groups who underwent fiberoptic bronchoscopy during the period from November 2007 to October 2008 at the Department of Cardiovascular and Thoracic Unit, Bir Hospital. Group I consisted of 35 cases of suspected lung malignancy on clinical and radiological examination, while group II consisted of 15 cases of non-neoplastic lung diseases.

Results: Bronchial brushing and forceps biopsy showed a sensitivity of 50\% and $84.21 \%$ respectively in the diagnosis of malignancy. Broncho-alveolar lavage revealed malignancy in $66.7 \%$ patients. Transthoracic needle aspiration had the highest sensitivity of $100 \%$ whereas bronchial wash was revealed malignancy only in $17.4 \%$ of the cases.

Conclusion: Combination of various cytohistological techniques complements each other and enhances the diagnostic efficacy of various neoplastic and non-neoplastic lung diseases. Bronchial brushing, forceps biopsy and transthoracic needle aspiration are complimentary in diagnosis of various lung malignancies whereas broncho-alveolar lavage is a useful procedure in detection of infections diseases.

\section{INTRODUCTION}

Lung cancer is currently the most frequently diagnosed and the most common cause of cancer mortality worldwide. ${ }^{1}$ In Nepal incidence of lung cancer has increased from 5.3\% in

\section{Correspondence:}

Dr. Anjani Tuladhar, $M D$

Department of Pathology, Kathmandu Model Hospital, Kathmandu, Nepal E-mail:anjanituladhar@hotmail.com
1989 to $7.3 \%$ in 1992 and $17.5 \%$ in $1996 .^{2}$ The increasing incidence could be due to increase in smoking habit, change in life styles of the people, increased environmental pollution and also the availability of different modern diagnostic modalities to detect lung cancer.

Similarly, pulmonary tuberculosis still remains a leading cause of death in developing countries and it is estimated 


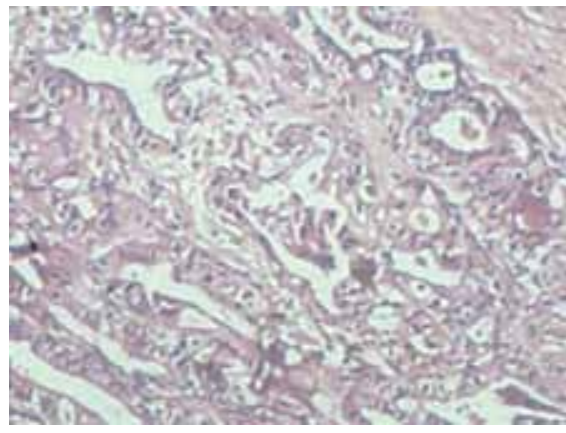

Figure 1: Adenocarcinoma showing atypical columnar cells in glandular pattern. (HE stain, X100)

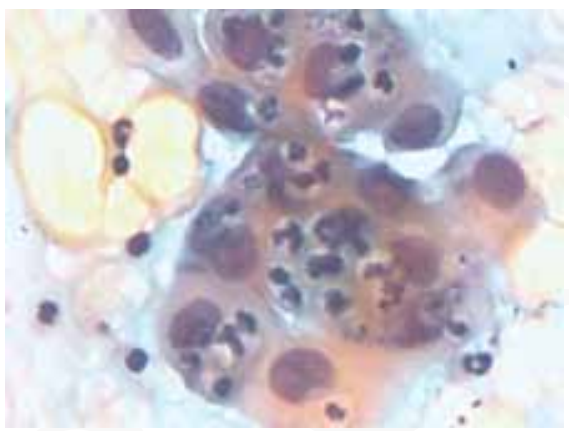

Figure 2: large cell carcinoma showing dispersed large pleomorphic cells and prominent neutrophil ingestion. (PAP stain, X400)

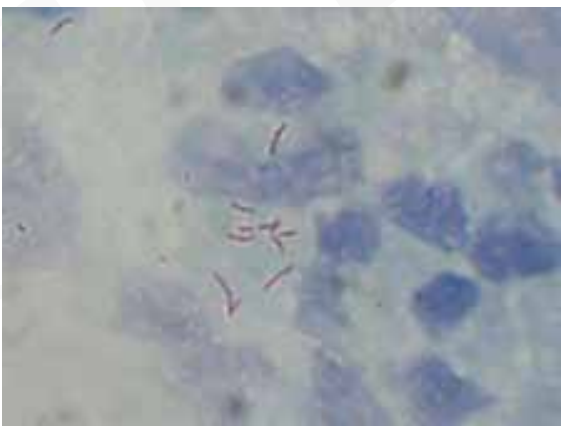

Figure 3: Clusters of acid-fast bacillus in BAL sample. (Ziehl Nelson stain, X1000) that 1.7 billion individuals are infected worldwide; with 8 to 10 million new cases and 3 million deaths per year. ${ }^{3}$ In Nepal estimated incidence is 176 per 100,000 populations according to data of $2006 .{ }^{4}$

An attempt has been made to determine whether a combination of various cytological methods is more effective than a single procedure in both neoplastic and nonneoplastic lung lesions.

\section{MATERIALS AND METHODS}

This prospective study was carried out in the departments of Cardiovascular and Thoracic Units and department of pathology of Bir Hospital. Total number of cases enrolled in the study was 50 and the study was conducted over a period of 12 months starting from November 2007 to October 2008. Based on clinical, radiological and Fiberopitc biopsy (FOB) findings, cases were divided into 2 groups - "neoplastic group" (group-I) and "non-neoplastic group" (group-II). Group I consisted of 35 cases of suspected lung malignancy on clinical and radiological examination, while group II consisted of 15 cases of non-neoplastic lung diseases.

One or more cytohistotechnique applied to diagnose lung lesions. In neoplastic group bronchial brush (BB) was carried out in 31 cases, bronchial wash (BW) in 30, bronchoalveolar lavage (BAL) in 6, transthoracic needle aspration (TTNA) in 4 and bronchial biopsy (FB) in 28 cases. Similarly, in non-neoplastic group BB was done in two cases, BW in one case and FOB in seven cases. BAL was done in almost all cases while TTNA was not done at all.

May-Grünwald-Giemsa (MGG) staining for air-dried smears and Papanicolaou (PAP) staining for alcohol-fixed smears were used. Ziehl-Neelsen (ZN) staining for acid fast bacilli (AFB) was done where needed. The biopsy material was formalin fixed and processed in histopathology laboratory. Sections of $3 \mathrm{~mm}$ thickness were cut by paraffin embedding technique. Hematoxylin and Eosin staining was done on tissue section for morphological evaluation.
Collected data was analyzed using SPSS13 and Microsoft Excel software.

\section{RESULTS}

The mean age of the patients with both neoplastic and non-neoplastic group was found to be $59.5 \pm 11.0$ years and $50.80 \pm 7.7$ years. There was a male preponderance in both neoplastic and non-neoplastic groups with $\mathrm{M}$ : $\mathrm{F}$ ratio of 4.3:1 and 3.7:1 respectively.

In the neoplastic group, FOB revealed growths in 23 cases $(65.7 \%)$, bleeding to touch in 15 cases $(42.8 \%)$, and narrowing in 12 cases $(34.2 \%)$. Increased secretions were seen in four cases $(11.4 \%)$. In the non-neoplastic group, FOB showed increased secretions in all cases (100\%) followed by bleeding to touch in 7 cases $(46.6 \%)$.

The various bronchogenic malignancies in decreasing order of frequency were squamous cell carcinoma $(51 \%)$, followed by small cell carcinoma (19\%), adenocarcinoma (11\%) (fig.1), nonsmall cell carcinoma (7\%), large cell carcinoma (4\%) (fig. 2), carcinoma in situ (4\%) and small round cell tumor $(4 \%)$.

Various cytohistotechnique were applied to diagnose lung cancer like BB, BW, TTNA and FOB. TTNA showed highest sensitivity $(100 \%)$ and proved helpful in cases which could not be diagnosed by other cytohistotechniques. FOB showed $84.2 \%$ sensitivity where as BW showed only $17.4 \%$ sensitivity. When $\mathrm{BB}$ and $\mathrm{BW}$ were combined the sensitivity increased upto $(59.1 \%)$ which was better than when either of them were considered singly. FOB showed the highest cell typing accuracy (92.5\%) with two cases categorized as non-small cell carcinoma which however could not be further subtyped. BAL showed better sensitivity $(66.7 \%)$ than $\mathrm{BB}$ and $\mathrm{BW}$ combined.

With the aid of various cytohistotechniques a variety of non-neoplastic lesions were diagnosed which included nonspecific inflammation followed by tuberculosis. Different non-neoplastic lesions are given in Table 1 . BAL proved to 
Table 1: Non-neoplastic lesions of lung (Group-II)

\begin{tabular}{ccc}
\hline Disease & No. of cases & \% \\
\hline Tuberculosis & 4 & 26.7 \\
\hline Abscess & 2 & 13.3 \\
\hline $\begin{array}{c}\text { Non-specific inflam- } \\
\text { mation }\end{array}$ & 8 & 53.3 \\
\hline Normal & 1 & 6.7 \\
\hline Total & $\mathbf{1 5}$ & $\mathbf{1 0 0}$ \\
\hline
\end{tabular}

be more efficacious than BB in the detection of tuberculosis. (fig.3)

\section{DISCUSSION}

\section{Neoplastic lung lesions}

Of 27 patients diagnosed to have lung cancer, 20 patients were males, with M: F ratio of 2.8:1. Out of these, 20 were smokers. The mean age of patients with lung cancer was $59.0+11$ years. A study done in Kathmandu, showed similar features with age range from 40 years to 78 years, with mean age of 59 years. Of the 42 cases with confirmed diagnosis, $33(78.59 \%)$ were males and $9(21.42 \%)$ were females with M: F ratio of 3.6:1.6

Squamous cell carcinoma (51\%) was the most common primary bronchogenic tumour in our study, followed by small cell carcinoma (19\%) and adenocarcinoma (11\%) as found in other studies. ${ }^{5,6}$ This is in contrast to studies in western countries, where incidence of adenocarcinoma has surpassed squamous cell carcinoma. ${ }^{7}$

Thirty-five patients (Group-I) who underwent FOB were studied; 27 of them were eventually found to have malignancy. Final diagnosis of cancer was achieved by any one or combination of these diagnostic modalities namely: $\mathrm{BB}, \mathrm{BW}, \mathrm{BAL}, \mathrm{FOB}$, radiologically-guided TTNA.

\section{Bronchial Brushing}

BB showed a diagnostic sensitivity of 50\% in detection of lung malignancy. Various studies done showed sensitivity of brushing to vary between $48-85.1 \% .^{8-11}$ The causes for varying sensitivity in previous studies include use of different techniques for the retrieval and processing of cytological specimens and inclusion of 'suspicious cases' as positive for calculation of sensitivity.

Garg $\mathrm{S}$ et $\mathrm{al}^{12}$ in their study found the sensitivity of bronchial brushing increased to $57.14 \%$ in cases with visible endobronchial abnormality which shows that $\mathrm{BB}$ is more sensitive in diagnosing central lesions.

In the present study, BB was found to be the most sensitive cytologic technique in diagnosing small cell carcinoma $(80 \%)$ followed by squamous cell carcinoma (35.7\%). Similar results were seen by Lundgren et $\mathrm{a}^{13}$ who found $90 \%$ sensitivity of BB in diagnosing small cell carcinoma and adenocarcinoma.

\section{Bronchial Wash}

In our study out of 30 cases, in five BW samples showed positive malignant cells (17.4\%). Mak et $\mathrm{al}^{14}$ found that combination of brushings and washings had a high additional sensitivity in visible tumors. They concluded that for the maximum diagnostic yield for the diagnosis of cancer, forceps biopsy should be combined with cytology using both washings and brushings, and believed that routine collection of either washings or brushings was costeffective.

\section{Bronchoalveolar Lavage}

BAL showed a sensitivity of $66.7 \%$ in diagnosing lung malignancy. The sensitivity varies between $14-76 \%$ in earlier studies reported. ${ }^{15-18}$ With BAL, due to less demonstrable cytological details, many samples were diagnosed as suspicious of malignancy.

\section{Bronchial Biopsy}

The diagnostic sensitivity of bronchial biopsy was $84.2 \%$. Previous reports have shown the sensitivity range from 65 $83 \%{ }^{19-22}$ The sensitivity of FOB in our study increased to $88 \%$ in cases having visible endobronchial abnormality. Similarly Funahansi et al got a higher sensitivity of $83 \%$ in visible endobronchial tumours. ${ }^{23}$

FOB showed $100 \%$ sensitivity in diagnosing small cell carcinoma followed by $92.85 \%$ in squamous cell carcinoma and $66.66 \%$ in adenocarcinoma. Similar results were seen by Buccheri et $\mathrm{al}^{24}$; who found $94 \%$ and $92 \%$ sensitivity of FOB in diagnosing squamous cell carcinoma and small cell carcinoma respectively.

Biopsy samples showed only $8.3 \%$ cases to be non-small cell (undifferentiated) carcinomas while rest could be specifically categorized as squamous, small cell or other types. There were 6 cases that did not yield adequate specimens from FOB. Similarly, 3 cases were not detected by biopsy and gave false negative results. Two of these false negative cases were reported as inflammatory lesions and one was reported as no obvious pathology present. Thus these lapses are due to sampling errors and due to not hitting the lesions at their core.

\section{TTNA}

TTNA was done in 4 patients and though this procedure is invasive there was no major complication. All 4 cases 
were positive for malignancies by this procedure, whereas only two of these cases were also reported malignant by other cytological techniques. So when other cytological techniques like BB and BAL was combined with TTNA the sensitivity was increased by $100 \%$.

The sensitivity of this procedure was $100 \%$ with a total predictive value of $50 \%$. Gasparini et $\mathrm{al}^{25}$ found a higher sensitivity of $93.2 \%$ by use of TTNA in diagnosis of peripheral pulmonary nodules. If the results were negative for malignancy and clinical suspicion was strong, the use of guided TTNA can be a valuable procedure for the diagnosis of these patients.

After several years of quiescence, pulmonary cytology enjoyed a period of rapid resurgence in the 1970s and 1980s, particularly as fine-needle aspiration (FNA) was validated as an alternative to open lung biopsy or transbronchial biopsy for the diagnosis of both neoplastic and non-neoplastic lung diseases. $^{25}$

No collection method is absolutely superior to the others. The choice of cell collection technique is shaped by factors such as the personal preference of the physician, the status of the patient, the location of the lesions, and the differential diagnosis. $^{25}$

\section{II.NON-NEOPLASTIC LUNG DISEASES}

The age group of the patients in non-neoplastic group ranged from 19 to 74 years with a mean of $50.80 \pm 7.7$ years in this study. Male to female ratio was 3.7:1. Shiner et al found the male to female ratio of 1:1 in non-neoplastic patients in their study. ${ }^{26}$

In our study 4 cases were suspected to have tuberculosis, out of which 2 showed positivity in BAL (fig.3). Other 2 negative cases were on therapeutic trial for tuberculosis and showed good response to the drugs. Baughman et $\mathrm{al}^{27}$ found a $50 \%$ sensitivity of BAL in the detection of the bacilli in 30 AFB smear-negative tuberculosis patients.

Of the 4 suspected cases of tuberculosis, two cases underwent FOB. In one case there was caseous necrosis. The reason for this might be inability to hit the lesion during biopsy. The sensitivity of BAL was better than biopsy which could be due to peripheral locations of the lesions which were better sampled by BAL and were inaccessible in biopsy.

The major value of bronchoscopy in these conditions is to obtain material for cytological and microbiological examination from patients who are unable to expectorate. Sometimes the procedure is done to rule out underlying lung malignancy and in patients who do not respond to empirical drug therapy.

Two cases were diagnosed as lung abscesses in our study and BAL cytology showed high neutrophil count $(91.5 \%)$ in these cases. Culture of the BAL specimens identified the causative organisms in both the patients. Bronchial biopsy done in one of the patients showed necrosis only.

Our study suggests that combination of various cytohistological procedures results in a statistically significant increase in the diagnostic yield for various neoplastic and non-neoplastic diseases of the lungs. BB, FOB and TTNA compliment each other in diagnosis and typing of various lung malignancies whereas BAL proved to be beneficial in detection of infectious diseases. TTNA is also a valuable procedure especially when all the cytological techniques yield no positive findings.

\section{CONCLUSION}

Thus a judicious combination of various cytohistotechniques i.e. BAL, BB, BW and FOB following FOB in the present study proved to be highly valuable in the diagnosis of various neoplastic and non-neoplastic lung diseases. In a minor subset of patients, where all the other cytohistotechniques proved illusive, TTNA proved to be highly sensitive and beneficial technique in diagnosing lung cancers.

\section{ACKNOWLEDGEMENT}

I would like to thank Dr. R.B.Basnet, NAMS, Bir Hospital for immense help preparing this article.

\section{REFERENCES}

1. Husain AN, Kumar V. The Lung. In: Robbins and Cotran. Pathologic basis of disease. 7th ed. Philadelphia: W.B. Saunders Company; 2006. pp711-22.

2. Shrestha HG, Dali S, Sayami G. Present cancer scenario and its changing pattern at TUTH. JNMA 1997;35:45-51.

3. Kumar V, Cotran RS, Robbins SL. Lungs and the upper respiratory tract. In: Basic pathology, 6th ed. Philadelphia: W.B. Saunders Company; 1999. pp393-43.

4. Recent news on Nepal from the weekly TB/malaria report. [Online]. 2006 [cited 2009 Feb 1]; Available from: URL:http://www. globalhealthreporting.org/countries/Nepal/

5. Chhajed PN, Athavale AV, Shah AC. Clinical and pathological profile of 73 patients with lung carcinoma: Is the picture changing? J Assoc physicians India 1999;47:483-7.

6. Jindal SK, Malik SK, Malik AK, Singh K, Gujral JS, Sodhi JS. Bronchogenic carcinoma (A review of 150 cases). Ind J Chest Dis All Sci 1979;21: 59-64.

7. Minna JD. Neoplasms of the lung. In : Braunwald E, Fauci AS, Kasper DL, Hauser SL, Longo DL, Jameson JL eds. Harrison's principles of internal medicine, 15th ed. New York: McGraw Hill company; 2001: pp562-71.

8. Bedrossian CM, Rybka DL. Bronchial brushing during fiberoptic bronchoscopy for the cytodiagnosis of lung cancer: comparison with sputum and bronchial washings. Acta Cytol 1976;20:446-53.

9. Jones AM, Hanson IM, Armstrong GR, O'Driscoll BR. Value and accuracy of cytology in addition to histology in the diagnosis of lung cancer at flexible bronchoscopy. Respir Med 2001;95:374-8. 
10. Chopra SK, Genovesi MG, Simmons DH, Gothe B. Fiberoptic bronchoscopy in the diagnosis of lung cancer. Comparison of pre and post-bronchoscopy sputa, washing, brushings and biopsies. Acta Cytol 1977;21:524-7.

11. Govert JA, Kopita JM, Matchar D, Kussin PS, Samuelson WM. Cost effectiveness of collecting routine cytologic specimens during fiberoptic bronchoscopy for endoscopically visible lung tumor. Chest 1996;109:451-6.

12. Garg S, Handa U, Mohan H, Janmeja AK. Comparative Analysis of Various Cytohistological Techniques in Diagnosis of Lung Diseases. Diagn. Cytopathol. 2007;35:26-31.

13. Lundgren R, Bergman F, Angstrom T. Comparsion of transbronchial fine needle aspiration biopsy, aspiration of bronchial secretion, bronchial washing, brush biopsy and forceps biopsy in the diagnosis of lung cancer. Eur J Respir Dis 1983;64:378-85.

14. Mak VHF, Johnston IDA, Hetzel MR, Grubb C. Value of washings and brushings at fiberoptic bronchoscopy in the diagnosis of lung cancer. Thorax 1990;45:373-6.

15. Semenzato G, Poletti V. Bronchoalveolar lavage in lung cancer. Respiration 1992;59 (Suppl):44-6.

16. Rennard SI. Bronchoalveolar lavage in the diagnosis of cancer. Lung 1990;168 (Suppl):1035-40

17. Linder J, Radio SJ, Robbins RA, Ghafouri M, Rennard SI. Bronchoalveolar lavage in the cytologic diagnosis of carcinoma of the lung. Acta Cytol 1987;31:796-801

18. Poletti V, Patelli M, Poggi S, Bertanti T, Spiga L, Ferracini R. Transbronchial lung biopsy and bronchoalveolar lavage in diagnosis of diffuse infiltrative lung diseases. Diagn Cytopathol 1988;54(s): 66-72.
19. Govert JA, Dodd LG, Kussin PS, Samuelson WM. A prospective comparison of fiberoptic transbronchial needle aspiration and bronchial biopsy for bronchoscopically visible lung carcinoma. Cancer 1999;87:121-34.

20. Karahalli E, Yilmaz A, Turker H, Ozvaran K. Usefulness of various diagnostic techniques during fiberoptic bronchoscopy for endoscopically visible lung cancer: Should cytologic examinations be performed routinely? Diagn Cytopathol 2001; 68:614-8.

21. Debeljak A, Mermolja M, Sorli J, Zupancic M, Zorman M, Remskar $\mathrm{J}$. Bronchoalveolar lavage in the diagnosis of peripheral primary and secondary malignant lung tumors. Respiration 1994;61:226-30.

22. Naryshkin S, Daniels J, Young NA. Diagnostic correlation of fiberoptic bronchoscopic biopsy and bronchoscopic cytology performed simultaneously. Diagn Cytopathol 1992;8:119-23.

23. Funahanshi A, Browne TK, Houser WC, Hranicka LJ. Diagnostic value of bronchial aspirate and post-bronchoscopic sputum in fiberotpic bronchoscopy. Chest 1979;76:514-17.

24. Buccheri G, Barbesis P, Delfino MS. Diagnostic, morphologic and histopathologic correlates in bronchogenic carcinoma: A review of 1,045 bronchoscopic examinations. Chest 1991;99:809-14.

25. Linder J. Lung Cancer cytology. Something old, something new. Am J Clin Pathol. 2000;14:169-71.

26. Shiner RJ, Rosenman J, Katz I, Reichert N, Hershko E, Yellin A. Bronchoscopic evaluation of peripheral lung tumors. Thorax 1988;43:887-9.

27. Baughman RP, Dohn MN, Loudon RG, Frame PT. Bronchoscopy with bronchoalveolar lavage in tuberculosis and fungal infections. Chest 1991;91:92-7. 